\title{
Article \\ The Fracture Behavior of 316L Stainless Steel with Defects Fabricated by SLM Additive Manufacturing
}

\author{
Hui $\mathrm{Li}^{1, * \mathbb{C}}$ and Jianhao Zhang ${ }^{2}$ \\ 1 Science and Technology on Reactor System Design Technology Laboratory, Nuclear Power Institute of China, \\ No. 328 Changshun Avenue, Chengdu 610200, China \\ 2 International Research Center for Computational Mechanics, State Key Laboratory of Structural Analysis for \\ Industrial Equipment, Faculty of Vehicle Engineering and Mechanics, Dalian University of Technology, \\ Dalian 116024, China; 1049066751@mail.dlut.edu.cn \\ * Correspondence: 1henpi@163.com
}

check for

updates

Citation: Li, H.; Zhang, J. The Fracture Behavior of 316L Stainless Steel with Defects Fabricated by SLM Additive Manufacturing. Crystals 2021, 11, 1542. https://doi.org/ $10.3390 /$ cryst11121542

Academic Editors: Ana Pilar Valerga Puerta, Severo

Raul Fernandez-Vidal, Zhao Zhang and Umberto Prisco

Received: 14 November 2021

Accepted: 7 December 2021

Published: 9 December 2021

Publisher's Note: MDPI stays neutral with regard to jurisdictional claims in published maps and institutional affiliations.

Copyright: (C) 2021 by the authors. Licensee MDPI, Basel, Switzerland. This article is an open access article distributed under the terms and conditions of the Creative Commons Attribution (CC BY) license (https:// creativecommons.org/licenses/by/ $4.0 /)$.

\begin{abstract}
In this paper, the fracture behaviors of 316L stainless steel with defects fabricated by the Selective Laser Melting (SLM) additive manufacturing are studied by a peridynamic method. Firstly, the incremental formulations in the peridynamic framework are presented for the elastic-plastic problems. Then, the pairwise force of a bond for orthotropic material model is proposed according to both the local and the global coordinate systems. A simple three-step approach is developed to describe the void defects that generated in the processing of the SLM additive manufacturing in the numerical model. Next, some representative numerical examples are carried out, whose results explain the validation and accuracy of the present method, and demonstrate that the orthotropic features, micro-cracks and voids of the materials have a significant influence on the ultimate bearing capacity, crack propagation and branching of the corresponding structures. It is also revealed that the crack initiations are induced actively by the defects and the crack branching is contributed to the complex multiple-crack propagation. Finally, the achievements of this paper lay a foundation for the engineering applications of the SLM additive manufacturing materials.
\end{abstract}

Keywords: peridynamic method; multiple-crack propagation; SLM additive manufacturing; defects

\section{Introduction}

Additive manufacturing techniques are on the basis of the design data and constitute a novel manufacturing method to produce solid structures by overlaying the raw materials automatically, which is attributed to its complex construction capability with high accuracy and good surface quality. At the same time, it cuts down greatly the time needed design and developing for the new products. Therefore, additive manufacturing techniques are promising to break through the limitations of traditional casting methods and provide the opportunity to realize the transformation and upgrading of the manufacturing industries [1-3]. According to different kinds of requirements, heat sources and materials, a great deal of additive manufacturing methods have been proposed, such as the Fused Deposition Modeling (FDM) method, the Electron Beam Melting (EBM) method, the Selective Laser Melting (SLM) method, and so on. Of these, it is pleasing that the SLM additive manufacturing method has great potential in the fields of aerospace, automotive and nuclear energy because of the good mechanical properties of the metal materials fabricated by this additive manufacturing method.

It is revealed that the process parameters of the SLM additive manufacture method, such as the layer thickness, the hatch spacing, the laser power, the scanning direction and speed, directly determine the temperature distribution, the grain growth, and the deformation of the corresponding materials [4]. Meanwhile, the defects of the micro-cracks and the voids are usually caused by the reasons of the nodulizing of the deposited metal, the high temperature gradient and the partially melted zone in the process, which has also 
attracted much attention from economists [5,6]. Until now, a lot of the related methods have been proposed to study the effects of different kinds of defects on the mechanical behaviors of the SLM additive manufacturing materials. For example, Zhang et al. [7] summarized the recent research outcomes on defect findings and classification in SLM, analyzed formation mechanisms of the common defects, such as porosities, incomplete fusion holes, and cracks. Jiang et al. [8] and Wu et al. [9] studied the effect of process parameters on defects, melt pool shape, microstructure and tensile behavior of the materials fabricated by the SLM. Zhang et al. [10] investigated the process parameters and post processing on fracture toughness and fatigue crack growth of the selective laser melting Ti-6Al-4V. Weber et al. [11] studied the fracture behavior and mechanical properties of a support structure for additive manufacturing of Ti-6Al-4V based on the experimental method. Glodez et al. [12] investigated the high-cycle fatigue and fracture behaviors of the selective laser melting SISi10Mg alloy with various kinds of defects. Wen et al. [13] developed a collective gray value method to automatically identify a class of defects of the additive manufacturing materials based on the different features of the defects in CT images. Lozanovski et al. [14] proposed a computational modelling for the mechanical analysis of the SLM manufactured lattice structures with hypothetical defects. In conclusion, the properties of the static strength, fatigue and fracture of the materials are significantly influenced by the defects of the micro-cracks and voids generated in the processing of the SLM additive manufacture, and extensive experimental methods have been usually applied to study these fracture behaviors. However, the experiments often have the disadvantages of long testing cycle and expensive cost, and the traditional numerical method is difficult to deal with the analysis of the complex fracture problems for the SLM fabricated materials with defects. Therefore, it is of great significance to develop an effective numerical method to study the complex fracture behaviors of materials with defects fabricated by SLM additive manufacturing.

In the most recent two decades, a non-local theory of the peridynamics proposed by Silling [15] has been continuously developed by many researchers [16-20], including the conventional bond-based model, the advanced ordinary/non-ordinary state-based model, and the dual-horizon model, etc. Meanwhile, peridynamics has been widely used in the mechanical fields [21-29]. Comparing with the continuum theory, the peridynamics is expressed by an integral equation in place of the differential equation, which is profitable to overcome the problems of the stress singularity at the tip of the cracks. For instance, Katiyar et al. [21,22] developed a peridynamic formulation for the pressure driven convective fluid transport in porous media with crack. Lai et al. [23,24] studied the geomaterial fragmentation by blast loads with the peridynamic method. Hu et al. [25] proposed a peridynamic model for dynamic fracture in unidirectional fiber-reinforced composites. Huang et al. [26-28] studied the quasi-static fracture and crack propagation problems of the brittle materials by an improved peridynamic approach. Oterkus and Madenci [29] presented a peridynamic model for the analysis of fracture problems in a fuel pellet. In a word, the peridynamics can easily handle the complex fracture problems, in which the crack path and branching are achieved to be a natural outcome of the simulation without any complicated numerical algorithms for crack path tracking. Consequently, the aim of this paper is to develop a peridynamic method for the analysis of the fracture behaviors of the SLM fabricated metal materials with defects, taking the 316L stainless steel for example.

This paper is organized as follows. Section 2 gives out the bond-based peridynamic formulations for the SLM additive manufactured orthotropic material and the fracture model. It also provides the way to present the pre-existing micro-cracks and voids. Section 3 shows several simple but illustrative numerical examples to verify the present method and to study the fracture behaviors of the 316L stainless steel with different kinds of defects. Finally, some conclusions are investigated in Section 4. 


\section{Peridynamic Model}

\subsection{Equation of Motion in Elastic-Plastic Theory}

With the bond-based peridynamic theory and the meshless method $[16,17]$, the domain of the interest is discretized into uniform material points, i.e., $\left\{\mathbf{x}^{i}, i=1,2,3, \cdots N\right\}, \mathbf{x}$ is the coordinate of the material point and $N$ is the total number of the material points. Then, the peridynamic expression of motion for any material point $\mathbf{x}^{i}$ can be expressed as

$$
\rho \ddot{\mathbf{u}}\left(\mathbf{x}^{i}, t\right)=\int_{H_{\mathbf{x}^{i}}} \mathbf{f}(\xi, \boldsymbol{\eta}) d V_{\mathbf{x}^{j}}+\mathbf{b}\left(\mathbf{x}^{i}, t\right)
$$

in which, $\rho$ is the mass density, $\ddot{\mathbf{u}}\left(\mathbf{x}^{i}, t\right)$ and $\mathbf{u}^{i}$ are the acceleration and displacement of material point $\mathbf{x}^{i}$ at time $t$, respectively. $H_{\mathbf{x}^{i}}=\left\{\mathbf{x}^{j} \mid\left\|\mathbf{x}^{j}-\mathbf{x}^{i}\right\| \leq \delta\right\}$ is the neighborhood of $\mathbf{x}^{i}$ with a horizon of $\delta, V_{\mathbf{x}}$ is the volume of $\mathbf{x}^{j}, \mathbf{b}\left(\mathbf{x}^{i}, t\right)$ is a prescribed body force density, and $\mathbf{f}(\xi, \boldsymbol{\eta})$ is a pairwise force (per unit volume squared) that the material point $\mathbf{x}^{j}$ exerts on the material point $\mathbf{x}^{i}$. The bond $\xi=\mathbf{x}^{j}-\mathbf{x}^{i}$ and $\boldsymbol{\eta}=\mathbf{u}^{j}-\mathbf{u}^{i}$ present the relative position and displacement of the material points $\mathbf{x}^{i}$ and $\mathbf{x}^{j}$ in the initial configuration and current configuration, respectively.

On the basis of the mechanical considerations of the conservation of linear and angular momenta, the pairwise force $\mathbf{f}(\xi, \boldsymbol{\eta})$ can be expressed as

$$
\mathbf{f}(\xi, \eta)=\underline{f} \frac{\xi+\eta}{\|\xi+\eta\|}
$$

To solve the elastic-plastic problems, the pairwise force is transferred into incremental form and the solutions are expressed as [18]

$$
\dot{f}= \begin{cases}c \bar{\omega}(\|\xi\|) \cdot \dot{s}, & \text { if }|\underline{f}|<f_{y} \text { or } \underline{f} \cdot \dot{s}<0 \\ 0, & \text { otherwise }\end{cases}
$$

where $c$ denotes the micro-modulus in a bond, $\bar{\omega}(\|\xi\|)$ is the weight function that presents the distribution of the force along the radius direction, the elongation of the bond $s$ is defined as

$$
s=\frac{\|\xi+\eta\|-\|\xi\|}{\|\xi\|}
$$

In addition, the value of pairwise force corresponding to the material yield can be obtained by [18];

$$
f_{y}= \begin{cases}\frac{2 \sigma_{y}}{A \delta^{2}}, & 1 D \\ \frac{12 \sigma_{y}}{\pi h \delta^{2}}, & 2 D \\ \frac{6 \sigma_{y}}{\pi \delta^{4}}, & 3 D\end{cases}
$$

where $A$ is the cross-sectional area, $h$ is the thickness, $\sigma_{y}$ is the yield stress of the material in continuum mechanics framework.

\subsection{Orthotropic Material and Fracture Model}

To consider the orthotropic characteristics of the materials fabricated by the SLM additive manufacturing, the orthotropic material model in peridynamics is improved in this section. Distinguishing from the traditional casting materials, the SLM additive manufacturing materials are built layer by layer on the basis of a planning scheme until it is complete, which leads the orthotropic features of the materials in mechanics. Meanwhile, the tensile strength along the laser scanning direction on a layer is greater than that along 
the orthotropic direction, that is the layer-to-layer orientation. Therefore, the pairwise force of a bond for the orthotropic material model can be expressed as

$$
\dot{\mathbf{f}}(\xi, \boldsymbol{\eta})= \begin{cases}\bar{\omega}(\|\xi\|) \cdot \dot{s} \mathbf{C H T}, & \text { if }|\underline{f}|<f_{y} \text { or } \underline{f} \cdot \dot{s}<0 \\ 0, & \text { otherwise }\end{cases}
$$

where $\mathbf{C}=\left[\begin{array}{cc}c^{\|} & 0 \\ 0 & c^{\perp}\end{array}\right]$ is the orthotropic elasticity matrix, $c^{\|}$and $c^{\perp}$ are the micro-moduli along the laser scanning direction on a layer and the layer-to-layer direction, respectively. $\mathbf{H}$ is a matrix for the coordinate transformation from the local coordinate system to the global coordinate system. $\mathbf{T}$ is a direction vector of the bond $\xi$ in the local coordinate system. As shown in Figure 1, $O^{\prime} x^{\prime} y^{\prime}$ is the local coordinate system that related to the laser scanning direction on a layer and $O x y$ is the global coordinate system. Thus, the coordinate transformation matrix $\mathbf{H}$ and the direction vector $\mathbf{T}$ can be written as

$$
\mathbf{H}=\left[\begin{array}{cc}
\cos \theta & -\sin \theta \\
\sin \theta & \cos \theta
\end{array}\right]
$$

and,

$$
\mathbf{T}=\left[\begin{array}{c}
\cos \alpha \\
\sin \alpha
\end{array}\right]
$$

in which, $\theta$ denotes the angle between the laser scanning direction on a layer and the $x$ axis in the global coordinate system, $\alpha$ denotes the angle between the bond $\xi$ and the $x^{\prime}$ axis in the local coordinate system.

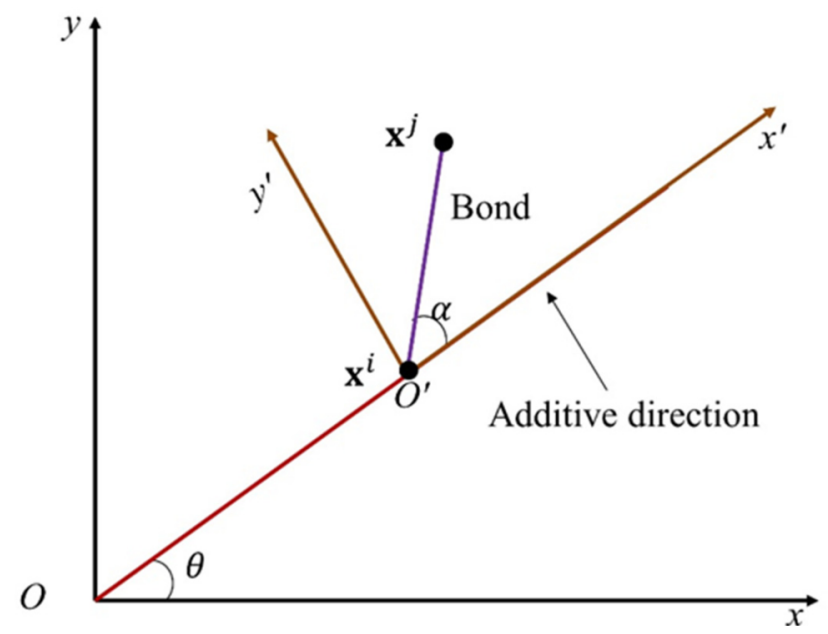

Figure 1. Schematic diagram of the local the global coordinate systems.

In addition, a scalar $\mu$ that used to define the failure state of the bond is introduced into Equation (6) to develop the fracture model in PD. Then, Equation (6) can be rewritten by

$$
\dot{\mathbf{f}}(\xi, \eta)= \begin{cases}\bar{\omega}(\|\xi\|) \cdot \dot{s} \mu \mathbf{C H T}, & \text { if }|\underline{f}|<f_{y} \text { or } \underline{f} \cdot \dot{s}<0 \\ 0, & \text { otherwise }\end{cases}
$$

where the scalar $\mu$ for the linear and bilinear material can be expressed as [28]

$$
\mu=\left\{\begin{array}{ccc}
1, & \mathrm{~s}<s_{0}, & \text { undamge } \\
1-d \frac{s_{\max }-s_{0}}{s_{\max }}, & \mathrm{s}<s_{\max } \text { and } s_{0}<s_{\max }<s_{\mathcal{c}}, & \text { unloading or reloading } \\
1-d \frac{s-s_{0}}{s_{\max }}, & \mathrm{s}=s_{\max } \text { and } s_{0}<s_{\max }<s_{\mathcal{c}}, & \text { maxstreth increasing loading } \\
0, & \mathrm{~s} \geq s_{\mathcal{c}}, & \text { failed bond }
\end{array}\right.
$$


in which $s_{0}$ and $s_{c}$ are the critical stretches of the bond at the linear elastic limit and at the failure state, respectively, and $s_{\max }$ is the maximum value of the stretch in the deformation history. $s_{c}$ can be obtained by the critical energy release rate of the material and is calculated by [30]

$$
s_{c}=\sqrt{\frac{G_{c}}{\left(\frac{6}{\pi} v+\frac{16}{9 \pi^{2}}(\kappa-2 v)\right) \delta}}
$$

where $G_{c}$ is the critical energy release rate, $\kappa$ and $v$ are the bulk modulus and shear modulus, respectively.

Further, a scaler field $\varphi\left(\mathbf{x}^{i}, t\right)$ is utilized to evaluate the failure state of the material, i.e.,

$$
\varphi\left(\mathbf{x}^{i}, t\right)=1-\frac{\int_{H_{\mathbf{x}^{i}}} \mu d V_{\mathbf{x}^{j}}}{\int_{H_{\mathbf{x}^{i}}} d V_{\mathbf{x}^{j}}}
$$

It can be seen from Equation (12) that the scaler field $\varphi\left(\mathbf{x}^{i}, t\right)$ is a damage factor and denotes the ratio between the broken bonds of the material point $x^{i}$ and the whole bonds in its neighborhood at time $t$. Therefore, the values of $\varphi\left(\mathbf{x}^{i}, t\right)$ are $0 \leq \varphi\left(\mathbf{x}^{i}, t\right) \leq 1$, with $\varphi\left(\mathbf{x}^{i}, t\right)=0$ presenting an undamaged state while $\varphi\left(\mathbf{x}^{i}, t\right)=1$ indicating a complete separate state.

\subsection{Characterization of Micro-Cracks and Voids}

In the processing of the SLM additive manufacturing, the elaborate mechanism comprising of the materials may result in the defects of the micro-cracks and voids due to the melting, solidification and phase transition, which has a great influence on the behaviors of the strength, fatigue and fracture of the materials. In this paper, on the basis of the shapes and sizes of the voids obtained by the measurement methods [13], the defects of the voids in the geometric model will be generated with a three-step approach:

Step 1: Discretize the whole region into material points with the meshless method.

Step 2: Generate the center coordinates of the voids with the uniform and random distribution methods according to the number and volume fraction of the voids in the whole region.

Step 3: Define the volume fraction of the voids $\Phi$ and the critical threshold $r$. Here, $r$ presents the distances between the centers of the voids and the material points. In the case of spherical voids, the material points will be removed when the distances between the material points and the center of sphere is less than the radius of the sphere.

In this paper, the fictitious defects are presented. Figures 2 and 3 show the void defects with different sizes and volume fractions using uniform and random distribution methods, respectively, in which the blue spots denote the void defects.
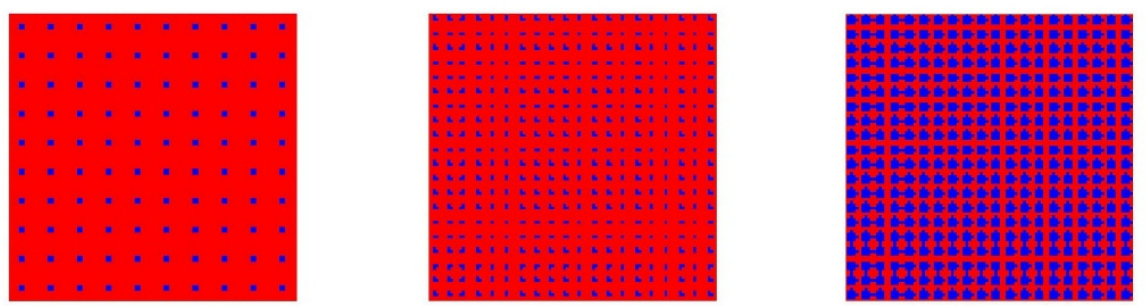

$r=0.01, \Phi=3.14 \% \quad r=0.01, \Phi=12.56 \% \quad r=0.02, \Phi=50.24 \%$

Figure 2. Uniform distribution void defects with different volume fractions. 


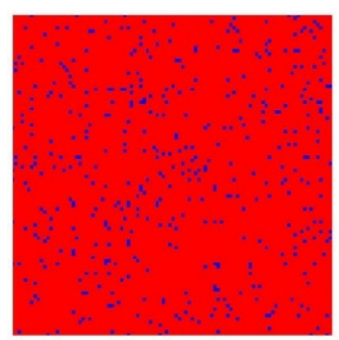

$\Phi=4.43 \%$

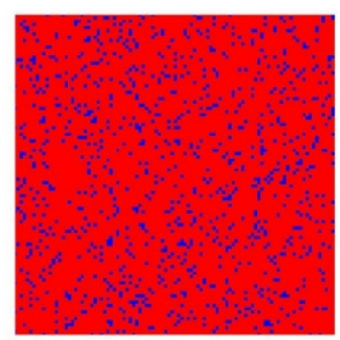

$\Phi=10.26 \%$

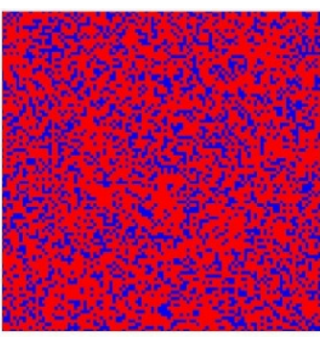

$\Phi=30.59 \%$

Figure 3. Random distribution void defects with different volume fractions.

\section{Numerical Examples}

A classical benchmark analyzed by Zaccaritto et al. [31] and Carpinteri and Colombo [32] is considered to verify the validation and accuracy of the present method for the analysis of the crack propagation problems. As shown in Figure 4, the curves of the reaction force versus the imposed displacement obtained by the present method fit well with the reference solutions and the overall slopes of the curves are well captured, which demonstrates the validation and accuracy of the present peridynamic method. Next, the proposed method will be used to analyze the effect of the pre-existing micro-crack, the orthotropic feature, and the void defects on the fracture behaviors of the SLM additive manufacturing 316L stainless steel.

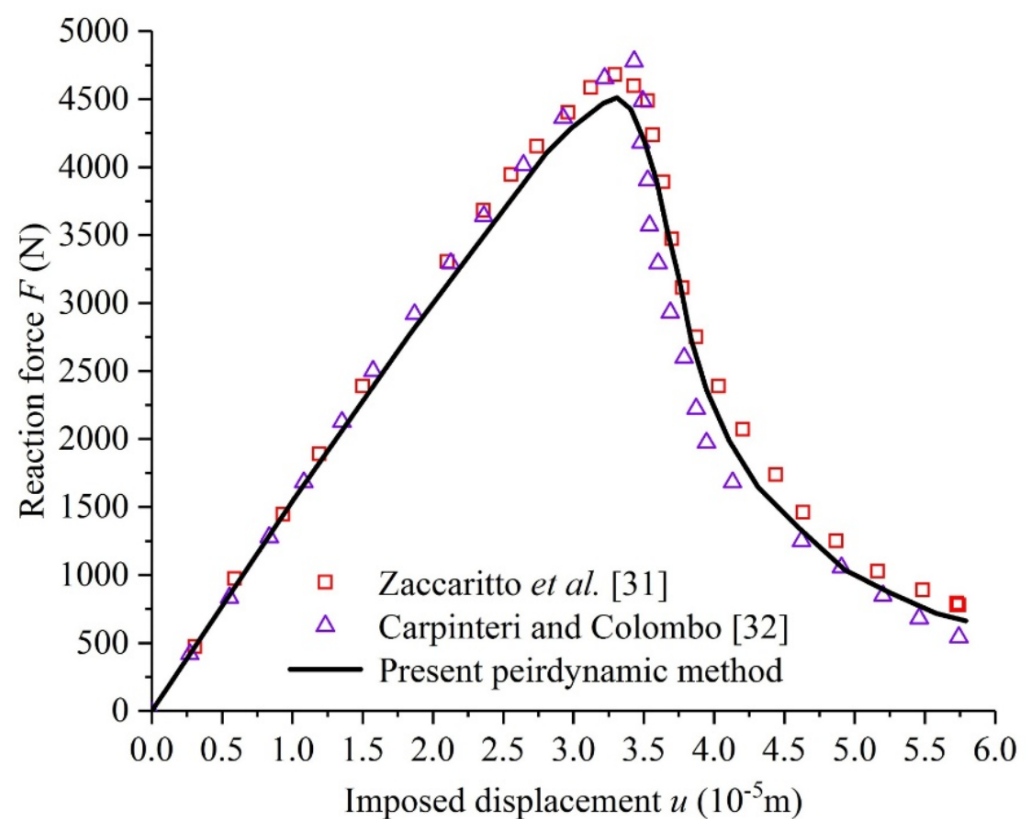

Figure 4. Curves of the reaction force versus imposed displacement obtained by the present method and reference solution.

\subsection{A Three-Point Bending Beam with a Pre-Existing Micro-Crack}

Firstly, a three-point bending beam with a pre-existing micro-crack is taken into account to study the effect of the location and size of the pre-existing micro-crack on the fracture behaviors of the SLM additive manufacturing 316L stainless steel. Figure 5 gives out the geometric dimensioning and the boundary conditions of the numerical model, in which the size of the beam is $1.0 \times 0.4 \times 0.0033 \mathrm{~m}^{3}$ with a vertical pre-existing notch at the bottom. The plane stress is assumed. For the spatial discretization, the beam is discretized into the uniform material points with the same sizes of $\Delta x=\Delta y=0.0033 \mathrm{~m}$ and the horizon of the neighborhood of the material points is $\delta=3 \Delta x$. For the material parameters, the Young's modulus is $E=2.12 \times 10^{11} \mathrm{~Pa}$, the Poisson's ratio is $v=0.3$, and the critical stretch of the bond is $s_{0}=0.01$. The velocity of the imposed loading is $10 \mathrm{~m} / \mathrm{s}$ 
and the time step of the integration is $1.0 \times 10^{-8} \mathrm{~s}$. Four kinds of pre-existing micro-cracks are considered: (1) $L=0, a=0.05 \mathrm{~m}$, (2) $L=0, a=0.1 \mathrm{~m}$, (3) $L=0.1 \mathrm{~m}, a=0.05 \mathrm{~m}$ and (4) $L=0.1 \mathrm{~m}, a=0.1 \mathrm{~m}$. Figure 6 plots the curves of the reaction force versus imposed displacement for different kinds of pre-existing micro-cracks, which explains that the location and size of the pre-existing micro-crack arising in the process of the SLM additive manufacturing will reduce the load-carrying limit of the corresponding structures. Figure 7 illustrates the crack patterns obtained by the present method at time $t=3.0 \times 10^{-4} \mathrm{~s}$ and $t=6.0 \times 10^{-4} \mathrm{~s}$ for these four loading cases, which shows that the pre-existing micro-crack also has a great influence on the path and velocity of the crack propagation.

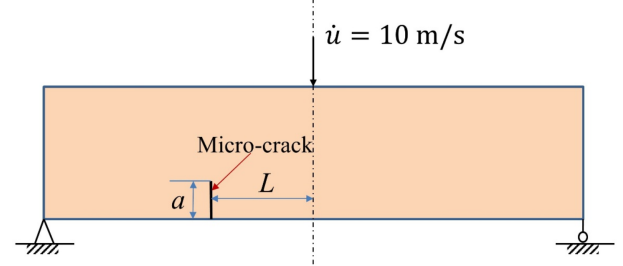

Figure 5. Schematic diagram of a three-point bending beam with a pre-existing micro-crack.

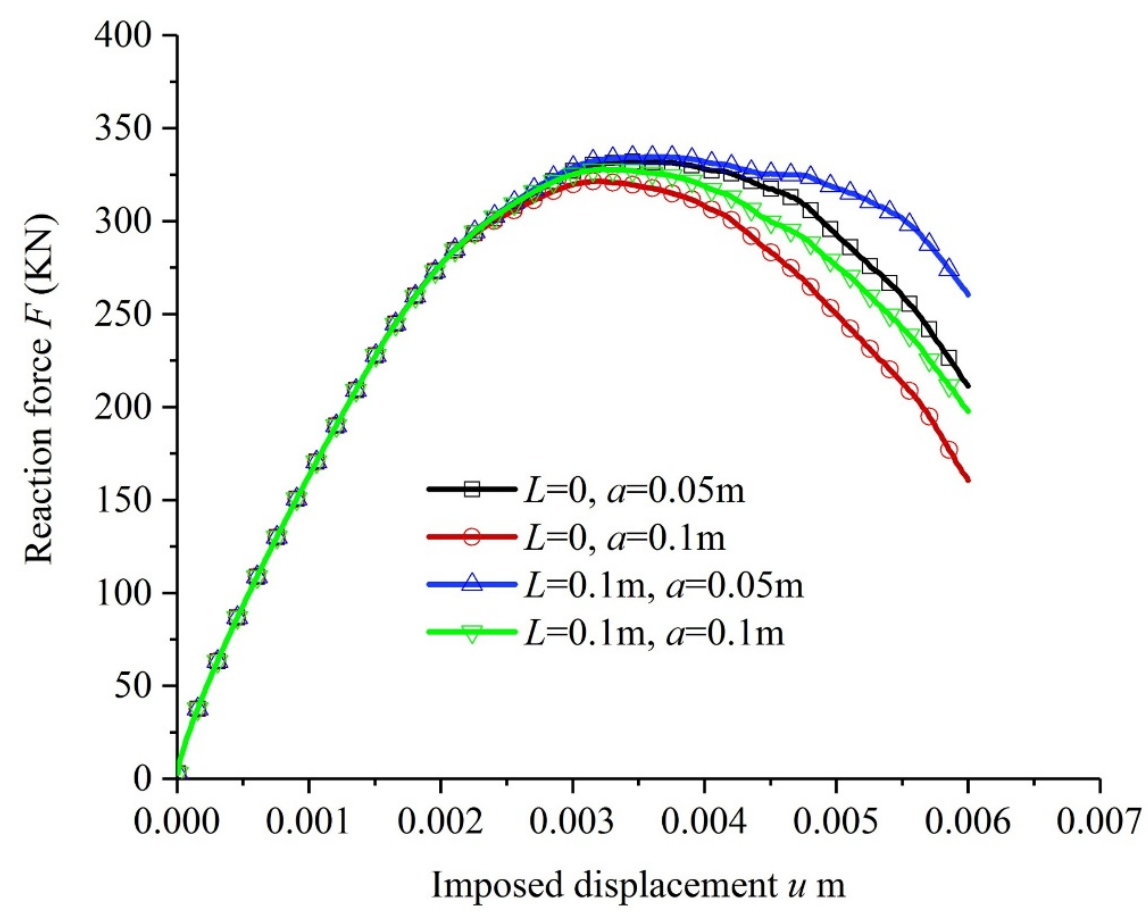

Figure 6. Curves of the reaction force versus imposed displacement for different kinds of pre-existing micro-cracks.
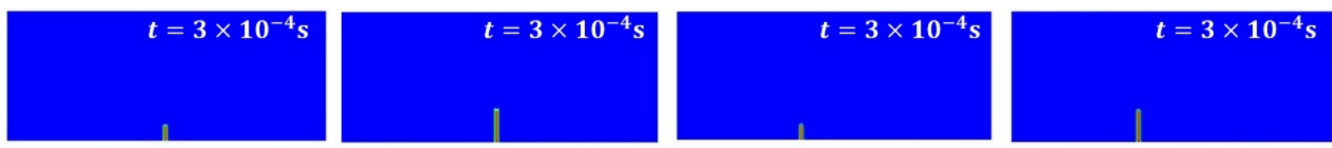

Damage
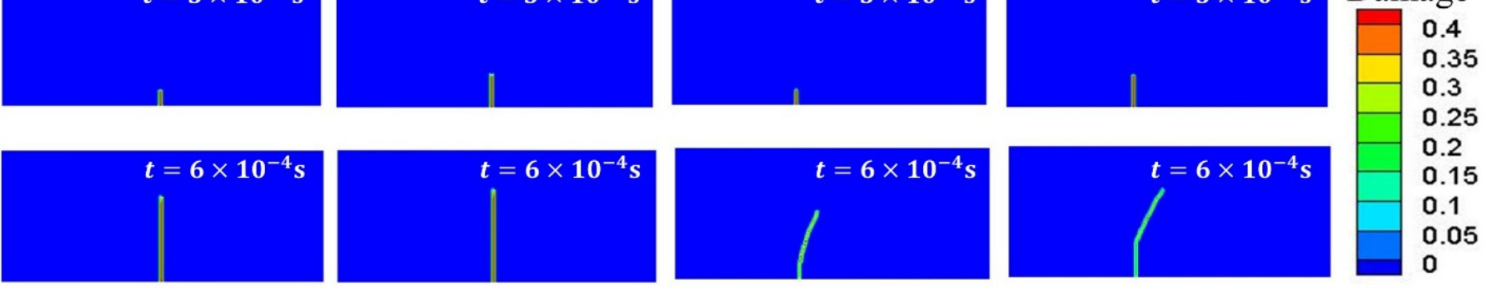

$L=0, a=0.05 \mathrm{~m}$

$L=0, a=0.1 \mathrm{~m}$

$L=0.1 \mathrm{~m}, a=0.05 \mathrm{~m}$

$L=0.1 \mathrm{~m}, a=0.1 \mathrm{~m}$

Figure 7. Crack patterns for these four loading cases at time $t=3.0 \times 10^{-4} \mathrm{~s}$ and $t=6.0 \times 10^{-4} \mathrm{~s}$. 


\subsection{A Three-Point Bending Beam with Orthotropic Moduli}

In this section, a three-point bending beam with orthotropic moduli, whose geometric and material parameters and whose boundary conditions are similar to those in Section 3.1, is considered to investigate the effect of the orthotropic features of the SLM additive manufacturing materials on their fracture behaviors. The initial micro-crack is $L=0.1 \mathrm{~m}, a=0.05 \mathrm{~m}$. Different kinds of ratios of the orthotropic Young's moduli, i.e., $\frac{E_{\perp}}{E_{\|}}=\lambda \leq 1.0$, are constructed, in which $E_{\|}=2.12 \times 10^{11} \mathrm{~Pa}$, the subscripts $\|$ and $\perp$ denote the laser scanning direction on a layer and the layer-to-layer direction, respectively. Figure 8 shows the curves of the reaction force versus the applied displacement for different ratios of the orthotropic Young's moduli. The results reveal that the smaller the Young's moduli ratios of the material (i.e., the Young's moduli along the layer direction is smaller), the smaller the load-carrying limit of the corresponding structure, which means that the performance of the additive manufactured material is related to the laser scanning strategy and the layer-to-layer bond strength. Figure 9 exhibits the contour plots of the crack propagation of the three-point bending beam at time $t=6.0 \times 10^{-4} \mathrm{~s}$, from which it is announced that the orthotropic features of the SLM additive manufacturing materials will also contribute to the path and velocity of the crack propagation.

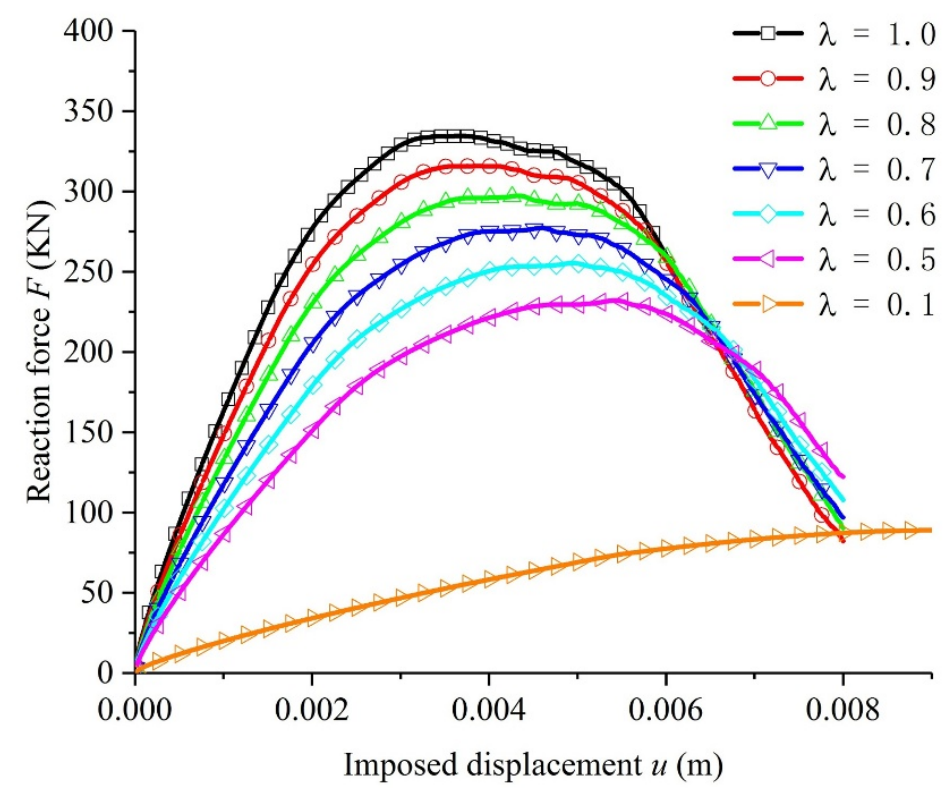

Figure 8. Curves of the reaction force versus imposed displacement for different ratios of the orthotropic Young's moduli.

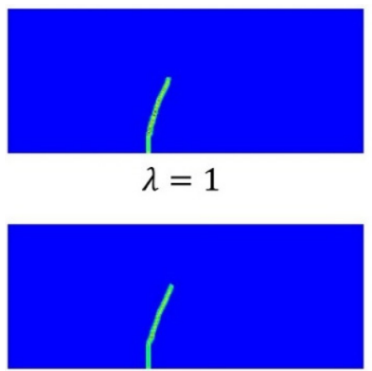

$\lambda=0.7$

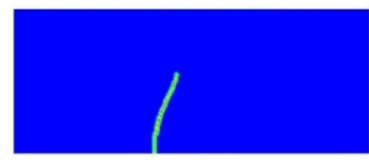

$\lambda=0.9$

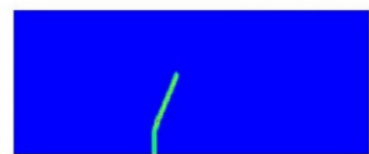

$\lambda=0.6$

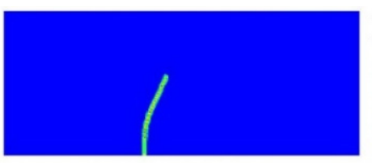

$\lambda=0.8$

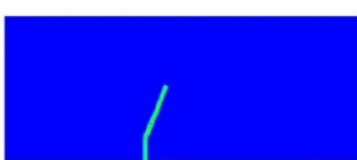

$\lambda=0.5$
Damage

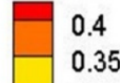

0.35

0.3

0.25

0.15

0.05

0

Figure 9. Contour plots of the crack paths for the additive manufactured orthotropic materials at time $t=6.0 \times 10^{-4} \mathrm{~s}$. 


\subsection{A Tensile Test of Plate Specimen with the Defects of Voids}

In order to analyze the impact of the defects of the micro-voids on the behaviors of the multiple crack growth for the SLM additive manufacturing 316L stainless steel, a tensile test of a plate specimen with random distribution defects of micro-voids is considered. The volume fraction of the micro-voids in the numerical model is $19.88 \%$. As shown in Figure 10, the size of the plate specimen is $0.4 \times 0.4 \times 0.002 \mathrm{~m}^{3}$ and the radius of two holes is $0.03 \mathrm{~m}$. The Young's modulus is $E=2.12 \times 10^{11} \mathrm{~Pa}$. The critical stretch $s_{0}$ of the bond is 0.01 . The interesting domain is discretized uniformly into $200 \times 200$ material points and the horizon $\delta$ of the neighborhood of the material point is $0.006 \mathrm{~m}$. The velocity of the imposed loading is $10 \mathrm{~m} / \mathrm{s}$ and the time step of the integration is $1.0 \times 10^{-8} \mathrm{~s}$. Figure 11 depicts the curves of reaction force versus imposed displacement obtained by the present method, from which it can be seen that the peak value of the reaction force for the case with random distribution defects is smaller that of the case with no defects, that is, the strength of the plate reduces because of the defects, and multiple-crack propagation will appear near the micro-voids. Furthermore, Figure 12 shows the paths of the multiple crack propagation of the plate specimen under tensile loading at time $t=0 \mathrm{~s}, t=0.8 \times 10^{-4} \mathrm{~s}$, $t=1.6 \times 10^{-4} \mathrm{~s}$ and $t=2.4 \times 10^{-4} \mathrm{~s}$. The results demonstrate that the micro-voids will actively induce the complex crack initiation and branching, which is contributed to the complex multiple crack propagation.

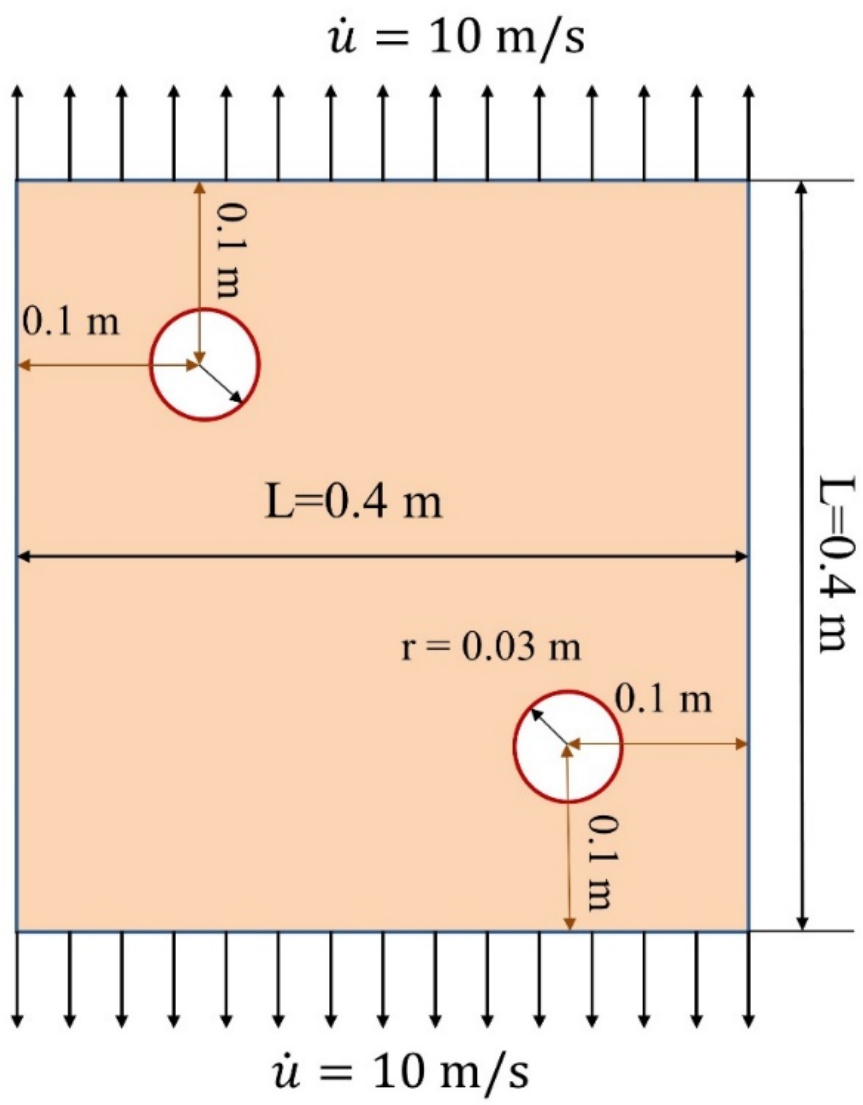

Figure 10. Schematic diagram of a tensile test of a plate specimen. 


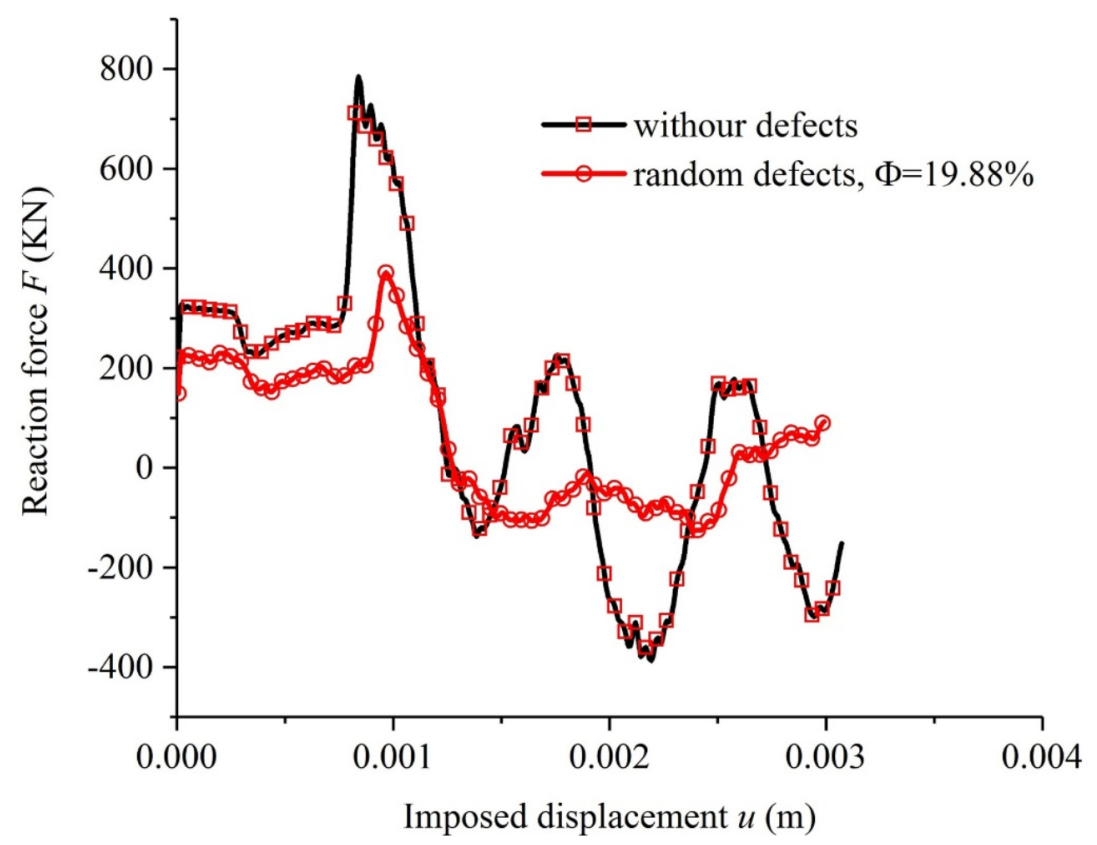

Figure 11. Curves of the reaction force versus imposed displacement.
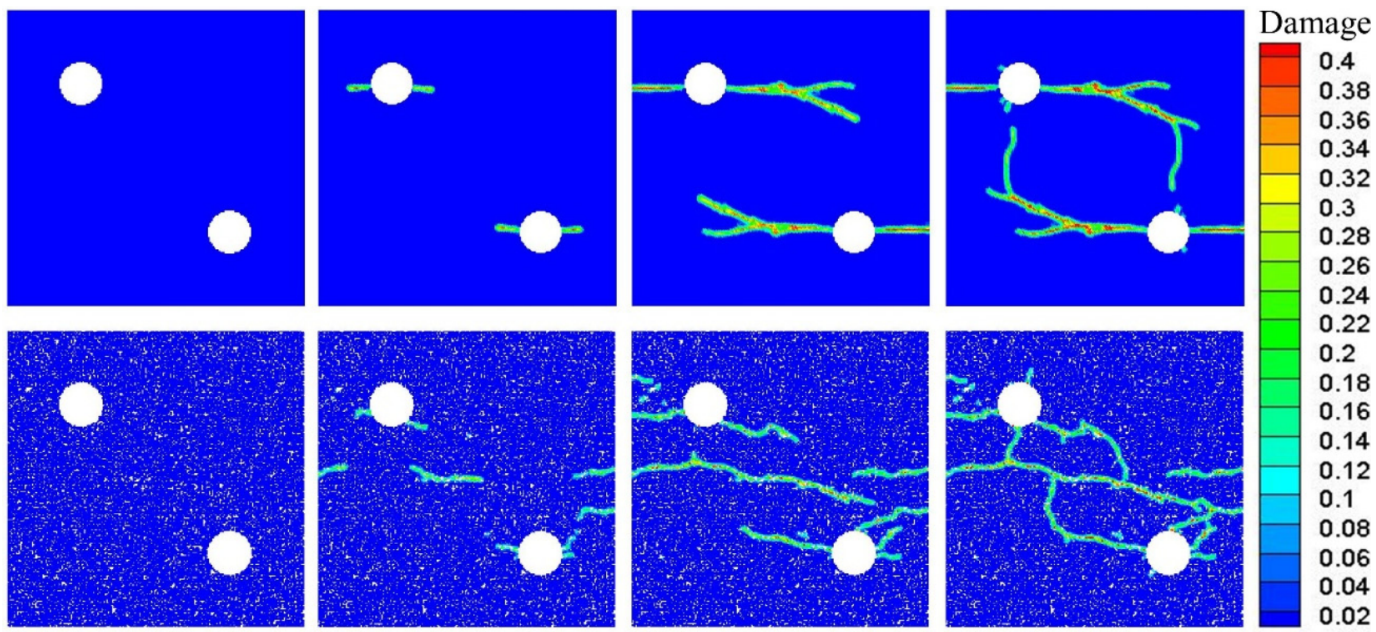

$t=1.6 \times 10^{-4} \mathrm{~S}$

$t=2.4 \times 10^{-4} \mathrm{~s}$

Figure 12. Paths of the multiple-crack propagation of the plate specimen under tensile loading at different time.

\section{Conclusions}

This paper presents a peridynamic method for the analysis of the fracture behaviors of 316L stainless steel with micro-cracks and voids fabricated by the SLM additive manufacturing. In this framework, the peridynamic formulation for the elastic-plastic theory was presented in incremental form. The pairwise force of a bond for the SLM additive manufacturing orthotropic material model was proposed according to the local and global coordinate systems. Then, a three-step approach was applied to describe the void defects in the geometric model, which was generated in the processing of the additive manufacturing. Next, three representative numerical examples were carried out and the results demonstrated that the present method can deal well with the complex multiple-crack problems of the additive manufacturing materials. Meanwhile, the results also indicated that the mechanical orthotropy, micro-cracks and voids had a significant influence on the ultimate bearing capacity, crack path and branching of the additive manufacturing materials. Finally, the achievements of this paper lay thew foundation for forming the 
evaluation methodology and criteria for the engineering applications of the SLM additive manufacturing materials.

Author Contributions: Conceptualization, H.L. and J.Z.; Formal analysis, H.L.; Methodology, H.L. and J.Z.; Validation, J.Z.; Writing-original draft, H.L.; Writing-review \& editing, J.Z. All authors have read and agreed to the published version of the manuscript.

Funding: This work was supported by the National Natural Science Foundation of China [No. 12102416].

Institutional Review Board Statement: Not applicable.

Informed Consent Statement: Not applicable.

Data Availability Statement: Not applicable.

Conflicts of Interest: The authors declare no conflict of interest.

\section{References}

1. Lu, B.H. Additive manufacturing-current situation and future. China Mech. Eng. 2020, 31, 19-23.

2. Zhang, X.J.; Tang, S.Y.; Zhao, H.Y.; Guo, S.Q.; Li, N.; Sun, B.B.; Chen, B.Q. Research status and key technologies of 3D printing. J. Mater. Eng. 2016, 44, 122-128.

3. Shamsaei, N.; Yadollahi, A.; Bian, L.; Thompson, S.M. An overview of direct laser deposition for additive manufacturing; Part II: Mechanical behavior, process parameter optimization and control. Addit. Manuf. 2015, 8, 12-35. [CrossRef]

4. Attar, H.; Bönisch, M.; Calin, M.; Zhang, L.C.; Scudino, S.; Eckert, J. Selective laser melting of in situ titanium-titanium boride composites: Processing, microstructure and mechanical properties. Acta Mater. 2014, 76, 13-22. [CrossRef]

5. Heigel, J.C.L.; Michaleris, P.; Reutzel, E.W. Thermo-mechanical model development and validation of directed energy deposition additive manufactureing of Ti-6Al-4V. Addit. Manuf. 2015, 5, 9-19.

6. Liu, X.B.; Xiao, H.; Xiao, W.J.; Song, L.J. Microstructure and crystallographic texture of laser additive manufactured nickel-based superalloys with different scanning strategies. Crystals 2021, 11, 591. [CrossRef]

7. Zhang, B.; Li, Y.; Bai, Q. Defect formation mechanisms in selective laser melting: A review. Chin. J. Mech. Eng. 2017, 30, 515-527. [CrossRef]

8. Jiang, H.Z.; Li, Z.Y.; Feng, T.; Wu, P.Y.; Chen, Q.S.; Feng, Y.L.; Chen, L.F.; Hou, J.Y.; Xu, H.J. Effect of process parameters on defects, melt pool shape, microstructure, and tensile behavior of 316 stainless steel produced by selective laser melting. Acta Metall. Sini. 2021, 34, 495-510. [CrossRef]

9. Wu, H.Y.; Zhang, D.; Yang, B.B.; Chen, C.; Li, Y.P.; Zhou, K.C.; Jiang, L.; Liu, R.P. Microstructural evolution and defect formation in a powder metallurgy nickel-based superalloy processed by selective laser melting. J. Mater. Sci. Technol. 2020, 36, 7-17. [CrossRef]

10. Zhang, H.Y.; Dong, D.K.; Su, S.P.; Chen, A. Experimental study of effect of post processing on fracture toughness and fatigue crack growth performance of selective laser melting Ti-6Al-4V. Chin. J. Aeronaut. 2019, 32, 2383-2393. [CrossRef]

11. Weber, S.; Montero, J.; Petroll, C.; Schäfer, T.; Blechmann, M.; Paetzold, K. The fracture behavior and mechanical properties of a support structure for additive manufacturing of Ti-6Al-4V. Crystals 2020, 10, 343. [CrossRef]

12. Glodez, S.; Klemenc, J.; Zupanic, F.; Vesenjak, M. High-cycle fatigue and fracture behaviours of SLM AlSi10Mg alloy. Trans. Nonferrous Met. Soc. China 2020, 30, 2577-2589. [CrossRef]

13. Wen, E.T.; Gao, T.T.; Zhang, Y.Y. 3D visualization method for complex lattice structure defects in 3D printing. Acta Metrol. Sin. 2020, 41, 1077-1081.

14. Lozanovski, B.; Leary, M.; Tran, P.; Shidid, D.; Ma, Q.; Choong, P.; Brandt, M. Computational modelling of strut defects in SLM manufactured lattice structures. Mater. Des. 2019, 171, 107671. [CrossRef]

15. Silling, S.A. Reformulation of elasticity theory for discontinuities and long-range forces. J. Mech. Phys. Solids 2000, 48, 175-209. [CrossRef]

16. Silling, S.A.; Lehoucq, R.B. Peridynamic theory of solid mechanics. Adv. Appl. Mech. 2010, 44, $73-168$.

17. Bessa, M.; Foster, J.T.; Belytschko, T.; Liu, W.K. A meshfree unification: Reproducing kernel peridynamics. Comp. Mech. 2014, 53, 1251-1264. [CrossRef]

18. Bobaru, F.; Foster, J.T.; Geubelle, P.H.; Silling, S.A. Peridynamic Modeling; Springer: New York, NY, USA, 2017.

19. Han, F.; Lubineau, G.; Azdoud, Y.; Askari, A. A morphing approach to couple state-based peridynamics with classical continnum mechanics. Comput. Methods Appl. Mech. Engrg. 2016, 301, 336-358. [CrossRef]

20. Ren, H.L.; Zhuang, X.Y.; Rabczuk, T. Dual-horizon peridynamics: A stable solution to varying horizons. Comput. Methods Appl. Mech. Eng. 2017, 318, 762. [CrossRef]

21. Katiyar, A.; Foster, J.T.; Ouchi, H.; Sharma, M.M. A peridynamic formulation of pressure driven convective fluid transport in porous media. J. Comput. Phys. 2014, 261, 209-229. [CrossRef]

22. Ouchi, H.; Katiyar, A.; York, J.; Foster, J.T.; Sharma, M.M. A fully coupled porous flow and geomechanics model for fluid driven cracks: A peridynamic approach. Comput. Mech. 2015, 55, 561-576. [CrossRef] 
23. Lai, X.; Ren, B.; Fan, H.F.; Li, S.F.; Wu, C.T.; Regueiro, R.A.; Liu, L.S. Peridynamics simulations of geomaterial fragmentation by impulse loads. Int. J. Numer. Anal. Methods Geomech. 2015, 39, 1304-1330. [CrossRef]

24. Fan, H.F.; Bergel, G.L.; Li, S.F. A hybrid peridynamics-SPH simulation of soil fragmentation by blast loads of buried explosive. Int. J. Impact Eng. 2016, 87, 14-27. [CrossRef]

25. Hu, W.; Ha, Y.D.; Boraru, F. Peridyanmic model for dynamic fracture in unidirectional fiber-reinforced composites. Comput. Methods Appl. Mech. Eng. 2012, 217, 247-261. [CrossRef]

26. Huang, D.; Lu, G.D.; Qiao, P.Z. An improved peridynamic approach for quasi-static elastic deformation and brittle fracture analysis. Int. J. Mech. Sci. 2015, 94, 111-122. [CrossRef]

27. Li, H.; Zhang, H.W.; Zheng, Y.G.; Ye, H.F.; Lu, M.K. An implicit coupling finite element and peridynamic method for the dynamic problems with crack propagation in solids. Int. J. Appl. Mech. 2018, 10, 1850037. [CrossRef]

28. Zhang, H.W.; Li, H.; Ye, H.F.; Zheng, Y.G. A coupling extended multiscale finite element and pridynamic method for modelling of crack propagation in solids. Acta Mech. 2019, 230, 3667-3692. [CrossRef]

29. Oterkus, S.; Madenci, E. Peridynamic modeling of fuel pellet cracking. Eng. Fract. Mech. 2017, 176, 23-37. [CrossRef]

30. Madeci, E.; Oterkus, E. Peridynamic Theory and Its Applications; Springer: New York, NY, USA, 2014.

31. Zaccariotto, M.; Luongo, F.; Sarego, G.; Galvanetto, U. Examples of applications of the peridynamic theory to the solution of static equilibrium problems. Aeronaut. J. 2015, 119, 677-700. [CrossRef]

32. Carpinteri, A.; Colombo, G. Numerical analysis of catastrophic softening behavior (snap-back instability). Comput. Struct. 1989, 31, 607-636. [CrossRef] 